\title{
Modeling of spatiotemporal patterns in bacterial colonies
}

\author{
A. M. Lacasta, I. R. Cantalapiedra, C. E. Auguet, A. Peñaranda, and L. Ramírez-Piscina \\ Departament de Física Aplicada, Universitat Politècnica de Catalunya, Avenida Dr. Marañon 44, E-08028 Barcelona, Spain
}

(Received 21 October 1998)

\begin{abstract}
A diffusion-reaction model for the growth of bacterial colonies is presented. The often observed cooperative behavior developed by bacteria which increases their motility in adverse growth conditions is here introduced as a nonlinear diffusion term. The presence of this mechanism depends on a response which can present hysteresis. By changing only the concentrations of agar and initial nutrient, numerical integration of the proposed model reproduces the different patterns shown by Bacillus subtilis OG-01. [S1063-651X(99)05706-2]
\end{abstract}

PACS number(s): 87.10.+e, 87.17.Aa, 47.54.+r

\section{INTRODUCTION}

Some kinds of bacterial colonies present interesting structures during their growth [1-11]. Depending on the bacterial species and the culture conditions, colonies can exhibit a great diversity of forms. In general, the complexity of the growth pattern increases as the environmental conditions become less favorable. Bacteria respond to adverse growth conditions by developing sophisticated strategies and higher microlevel organization in order to cooperate more efficiently. Examples of these strategies are the differentiation into longer-motile bacteria, the production of extracellular wetting fluid, the secretion of surfactants which change the surface tension, or the chemotactic response to chemical agents produced by bacteria [1-8]. The experiments are usually made in a Petri dish, which contains a solution of nutrient and agar. A drop of bacterial solution is then inoculated in the center of the dish. The growth conditions are controlled by the initial concentration of the medium components. The agar concentration determines the consistency of the medium, which becomes harder as the amount of agar increases, and the nutrient concentration controls the bacterial reproduction. Depending on these two factors, the colony grows at a higher or lower rate, developing different kinds of patterns.

In particular, colonies of the bacterium Bacillus subtilis OG-01 present a rich variety of structures [8-11]. Figure 1 shows the morphological diagram obtained by Ohgiwara, Matsushita, and Matsuyama [10]. They classified the colony patterns into five types, from $A$ to $E$, whose main features can be summarized as follows. If the medium is very hard, i.e., with a high concentration of agar, bacteria can hardly move and the colony essentially grows due to the consumption of nutrient and subsequent reproduction. If the level of nutrient is also low (region $A$ ), the growth is controlled by the diffusion of the nutrient up to the bacteria placed at the interface. The colony develops a ramified structure very similar to the patterns obtained with the diffusion-limited aggregation model (DLA) $[9,12]$. It takes approximately 1 month to cover the dish. If the initial agar concentration remains high and the nutrient concentration is increased, the growth is faster than in region $A$. The branches grow thicker until they fuse into a dense disk with rough interface (region $B)$, similar to the patterns obtained with an Eden model [13].
This structure needs 5-7 days to cover the disk. When the level of agar is decreased, which produces a medium a little softer than in region $B$, and the level of nutrient remains high, the colony forms concentric rings (region $C$ ). This region is characterized by periodic dynamics: for $2-3 \mathrm{~h}$ the colony expands while the bacteria move actively ("migration phase") and then they almost stop for 2-3 h ("consolidation phase",), during which the colony does not grow appreciably and the bacterial density increases due to reproduction. The crossover between the two phases is sharp. The periodic cycles of subsequent migration and consolidation phases create the pattern of concentric rings $[8,11]$. Accurate measurements show that in the growth phase there is a high concentration of longer and more motile bacteria, as a consequence of a differentiation process [8]. When a high level of nutrient is maintained, and the agar concentration is decreased further, the colony spreads over the agar plate, and after less than $8 \mathrm{~h}$ a homogeneous disk of low bacterial density is formed covering all the dish (region $D$ ). In this thin surface, bacteria are always short and can move easily by swimming. By decreasing the nutrient concentrations for a

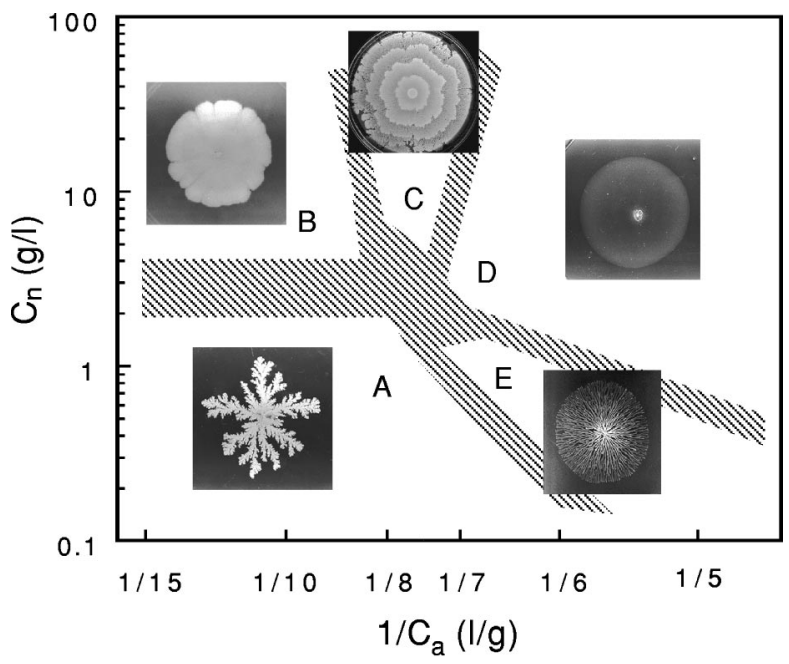

FIG. 1. Morphological diagram of patterns observed in colonies of Bacilus subtilis OG-01 as a function of nutrient concentration $\left(C_{n}\right)$ and the hardness of agar surface $\left(1 / C_{a}, C_{a}\right.$ being the agar concentration). Experiments were performed in a Petri dish with a diameter of $88 \mathrm{~mm}$. Taken with permission from [8]. 
semisolid medium, the colony develops a densely branched pattern (region $E$ ) similar to the dense branching morphology (DBM) found in other systems [14]. The ratio of the width of the branches to the gap between them is constant over the whole colony. The colony grows quite fast, showing its main activity at the tips of the fingers, and covering the dish in less than $24 \mathrm{~h}$. The dynamics is related to both the consumption of nutrients and the bacterial motility. In general, when environmental conditions are adverse (low nutrient or hard surface), a higher level of cooperation is observed.

The existence of a cooperative behavior seems to be determinant in the formation of the rings patterns of region $C$. The same kind of concentric rings has been found in experiments with other bacterial species. In the case of the bacterium Proteus mirabilis, the migration phases clearly involve the movement of differentiated swarmer bacteria (elongated and hyperflagellated) [5-7]. Similar ring patterns have also been observed in other nonliving systems, like the Liesegang rings produced by precipitation in the wake of a moving reaction front [15] or some experiments of interfacial electrodeposition [16]. In the case of the Liesegang patterns, it is well known that the distance between rings increases as $t^{1 / 2}$, whereas in bacterial and electrodeposition it is constant.

Several models have been proposed to explain the variety of patterns exhibited by Bacillus subtilis, as shown in Fig. 1 [2,3,9,17-21]. DLA-like patterns (region $A$ in Fig. 1) have been interpreted [9] as growth controlled by the diffusion of nutrients in the context of the DLA model. Ben-Jacob and co-workers proposed a communicating walkers model to describe some of the morphologies [2,3]. This model reproduces the crossover between regions $A$ and $B$ by coupling random walkers to fields representing the nutrients. DBMlike patterns are also obtained by introducing a chemotactic agent. Other kinds of models are based on reaction-diffusion equations for bacterial density. The Fisher equation [17] can be used for reproducing the homogeneous circular morphology (region $D$ ) [18]. Further developments were achieved by introducing new elements to the Fisher model, such as a field for nutrient and nonlinear diffusion coefficients $[19,20]$. Depending on the new elements introduced, these models reproduce some of the patterns of Fig. 1. However, the ring patterns (region $C$ ) have so far eluded a satisfactory modelization. Although the model suggested in Ref. [20] can generate concentric ring patterns, they are rather different from those observed in experiments [8]. In fact, dynamical cycles of consolidation and growth phases are not found. Finally, we must mention a model proposed by Esipov and Shapiro [21] for the study of Proteus mirabilis colonies, which introduces a lifetime for the differentiated swarmer bacteria. This model reproduces concentric ring patterns but does not explain why no periodicity is observed in other regions of the morphological diagram.

In this paper, we propose a model consisting of two coupled diffusion-reaction equations for bacteria and nutrient concentrations, where the bacterial diffusion coefficient can adopt two different expressions, corresponding to two possible mechanisms of motion. The first is the usual random swimming performed by bacteria in a liquid medium. The second is developed by bacteria in response to adverse growth conditions, and depends on their concentration. Bac- terial response is modeled as a global variable that can present hysteresis. Our model reproduces the five morphologies observed in the experiments, including the ring patterns.

\section{MATHEMATICAL MODEL}

We consider a two-dimensional system containing bacteria and nutrients, both diffuse, while bacteria proliferate by feeding on nutrient. Let us denote by $\bar{b}\left(\mathbf{r}^{\prime}, \tau\right)$ the density of bacteria at time $\tau$ and spatial position $\mathbf{r}^{\prime}$, and by $\bar{n}\left(\mathbf{r}^{\prime}, \tau\right)$ the concentration of nutrient. Then, $\bar{b}$ and $\bar{n}$ are in general governed by the following equations $[17,19]$ :

$$
\begin{gathered}
\frac{\partial \bar{b}}{\partial \tau}=\nabla D_{b} \nabla \bar{b}+\theta f(\bar{b}, \bar{n}), \\
\frac{\partial \bar{n}}{\partial \tau}=D_{n} \nabla^{2} \bar{n}-f(\bar{b}, \bar{n}) .
\end{gathered}
$$

The function $f(\bar{b}, \bar{n})$ denotes the consumption term of nutrient by bacteria, and can be described by Michaelis-Menten kinetics [17]

$$
f(\bar{b}, \bar{n})=\frac{k \bar{n} \bar{b}}{\left(1+\gamma^{\prime} \bar{n}\right)}
$$

where $k$ is the intrinsic consumption rate. For small $\bar{n}$, the consumption rate is approximately linear in $\bar{n}$ and it saturates at the value $k / \gamma^{\prime}$ as $\bar{n}$ increases. $D_{b}$ and $D_{n}$ are the diffusion coefficients of bacteria and nutrient, respectively. We assume that $D_{n}$ is constant, but $D_{b}$ can depend on nutrient and bacterium concentrations.

As explained above, experiments show that in adverse conditions, bacteria can adapt themselves in order to improve their motility. In a soft medium and high nutrient concentration (region $D$ of Fig. 1), short bacteria can swim randomly without difficulty, but in an adverse environment (regions $A$, $B, C$, and $E$ ) they need to develop mechanisms to become more motile. For intermediate conditions of semisolid medium and sufficient nutrient, there are periods of fast growth (migration phase) and slow growth (consolidation phase) that lead to the concentric ring patterns.

The analysis of periodic rings suggests a dynamical scheme with hysteresis that can be outlined in the following way: during the consolidation phase, the population of longer-motile bacteria increases in order to overcome the opposition to the movement. When this population exceeds a certain value, enhanced movement becomes possible and a migration phase begins. Then, however, a progressive decrease in long-bacterial population ensues, until it reaches a minimum at which the "enhanced-movement mechanism" does not work. Then a new consolidation phase begins. Within this scheme, region $D$ corresponds to a case where the maximum value is never reached (and therefore bacteria always move by the usual diffusion), whereas in regions $A$, $B$, and $E$ long-bacterial population does not fall below the minimum (and therefore always moves by the enhancedmovement mechanism). All these ideas can be introduced in our model by means of two basic points. 
(a) The diffusion coefficient $D_{b}(\bar{n}, \bar{b})$ can take two different expressions depending on the long-bacterial population.

(b) The net production of long bacteria depends on the environmental conditions and also on the colony phase of growth.

According to these ideas, we propose the following function for $D_{b}$ :

$$
D_{b}=D\left(\bar{d}_{1}+\bar{d}_{2} \bar{b}\right) \bar{n}
$$

where $D$ depends on the concentration of agar, which is lower for a harder medium. To take into account the inhomogeneities of the medium, we introduce a quenched disorder in $D$, which is written as $D=D_{0}\left[1+\xi\left(\mathbf{r}^{\prime}\right)\right], \xi\left(\mathbf{r}^{\prime}\right)$ being a random term defined on a square lattice. From now on, $D_{0}$ will be referred to as the diffusion parameter.

The first term of Eq. (3) describes the usual diffusion of bacteria in a liquid medium. The second describes the cooperative enhanced-movement mechanism promoted by long bacteria. This second mechanism can be modeled by a diffusion coefficient that depends on the bacterial concentration. We multiply both terms by nutrient concentration to take into account the fact that bacteria are inactive in the region where nutrient has been depleted. This dependence on $\bar{n}$ would not have been necessary if we had considered a "death" term in the equation for $\bar{b}$. The coefficients $d_{1}$ and $d_{2}$ can adopt two different values (one of them zero) depending on the concentration of the long bacteria, as will be specifed below.

Equations (1) with Eqs. (2) and (3) can be written in a simpler form as

$$
\begin{gathered}
\frac{\partial b}{\partial t}=\nabla\left\{D\left(d_{1}+d_{2} b\right) n \nabla b\right\}+\frac{n b}{1+\gamma n}, \\
\frac{\partial n}{\partial t}=\nabla^{2} n-\frac{n b}{1+\gamma n},
\end{gathered}
$$

with

$$
\begin{gathered}
\mathbf{r}=\left(\frac{\theta k^{2}}{D_{n}}\right)^{1 / 4} \mathbf{r}^{\prime}, \quad t=k\left(\theta D_{n}\right)^{1 / 2} \tau, \\
n=\left(\frac{\theta}{D_{n}}\right)^{1 / 2} \bar{n}, \quad b=\left(\frac{1}{\theta D_{n}}\right)^{1 / 2} \bar{b}, \\
\gamma=\left(\frac{D_{n}}{\theta}\right)^{1 / 2} \gamma^{\prime}, \quad d_{1}=\left(\frac{1}{\theta D_{n}}\right)^{1 / 2} \bar{d}_{1}, \quad d_{2}=\bar{d}_{2} .
\end{gathered}
$$

At this point, we need to specify how to choose $d_{1}$ and $d_{2}$ depending on the population of long bacteria. In order to do this, we introduce a global phenomenological quantity $W(t)$ that measures the amount of long bacteria. The evolution of this quantity should have a "creation term" that represents the transformation of short bacteria into long ones and an "annihilation term" that represents the opposite transformation (septation). It seems reasonable to assume that the creation term is directly dependent on the mean bacterial concentration, and inversely dependent on the level of nutrient $\left(n_{0}\right)$ and on the diffusion parameter (adverse conditions, i.e.,
$D_{0}$ and $n_{0}$ small, mean a faster differentiation process). With regard to the annihilation term, it can adopt two possible values depending on the growth phase. The simplest equation that includes all these considerations can be written as

$$
\frac{\partial W}{\partial t}=\lambda \frac{B}{n_{0} D_{0}}-c_{i},
$$

where $\lambda$ is a constant and $c_{i}$ can have two different values ( $c_{g}$ or $c_{s}, c_{g}>c_{s}$ ). The quantity $B$, defined as $B=\Sigma b^{2} / \Sigma b$, is a measure of the mean concentration of bacteria inside the colony. We introduce the hysteresis previously pointed out by assuming that there are two limit values $W_{\max }$ and $W_{\min }$ for which

$$
\begin{aligned}
& \text { when } \quad W \geqslant W_{\max } \text { then } \quad c_{i}=c_{g}, \quad d_{2}=D_{2}, \quad d_{1}=0, \\
& \text { when } \quad W \leqslant W_{\min } \text { then } \quad c_{i}=c_{s}, \quad d_{2}=0, \quad d_{1}=1 .
\end{aligned}
$$

With a suitable choice of parameters $\lambda, c_{s}$, and $c_{g}$, and by changing only $n_{0}$ and $D_{0}$, we can obtain colonies that always move with one of the two types of diffusion or colonies that periodically change from one type to the other. This will occur if $c_{s}<\lambda B /\left(n_{0} D_{0}\right)<c_{g}$, which will give rise to the ring patterns. Although the bacterial response has been expressed in terms of the population of long bacteria, other possible kinds of responses admit identical modelization. In this sense, our model is quite general.

\section{NUMERICAL RESULTS}

We have numerically integrated Eqs. (4) with Eqs. (6) and (7) in a square lattice of lateral size $L=600$ using a fourthorder Runge-Kutta method with mesh size $\Delta x=0.5$ and time step $\Delta t=0.005$. The system was initially prepared by assigning to each point a nutrient concentration $n(\mathbf{r}, 0)=n_{0}$ $+\eta(\mathbf{r}, 0), \eta$ being a uniform random number in the interval $(-0.1,0.1)$, and a bacterial concentration $b(\mathbf{r}, 0)=0$, except in a small central square where $b(\mathbf{r}, 0)=b_{0}$. The random term of the diffusion, $\xi(\mathbf{r})$, takes a different and uncorrelated value in each box of side $4 \Delta x$. The random values are assumed to be uniformly distributed in the interval $(-\epsilon, \epsilon)$. The box size and the intensity $\epsilon$ do not essentially affect the results.

In all our simulations, we used the parameters $b_{0}=0.7$, $\gamma=0.5, D_{2}=30, \epsilon=0.4, \lambda=0.18, c_{g}=2, c_{s}=1.6, W_{\max }$ $=3$, and $W_{\min }=2$. We reproduce the different morphologies observed in experiments by changing the values of the initial concentration of nutrients $n_{0}$ and the softness of the media, related to $D_{0}$.

In Figs. 2 and 3 we present the results obtained for $D_{0}$ $=0.005$ and different values of $n_{0}$. By increasing $n_{0}$ we reproduce the crossover between regions $A$ and $B$ of Fig. 1, from DLA-like patterns [Fig. 2(a)] to a dense rough structure similar to that found with an Eden model [Fig. 2(b)]. All of them correspond to a situation in which, due to the small value of $D_{0}$, the creation term of Eq. (6) is greater than $c_{g}$, except at the very beginning. The response $W$ can never decrease below the value $W_{\text {min }}$, and therefore the colony will always grow with the enhanced-movement mechanism. In spite of this cooperative mechanism, and because of the 

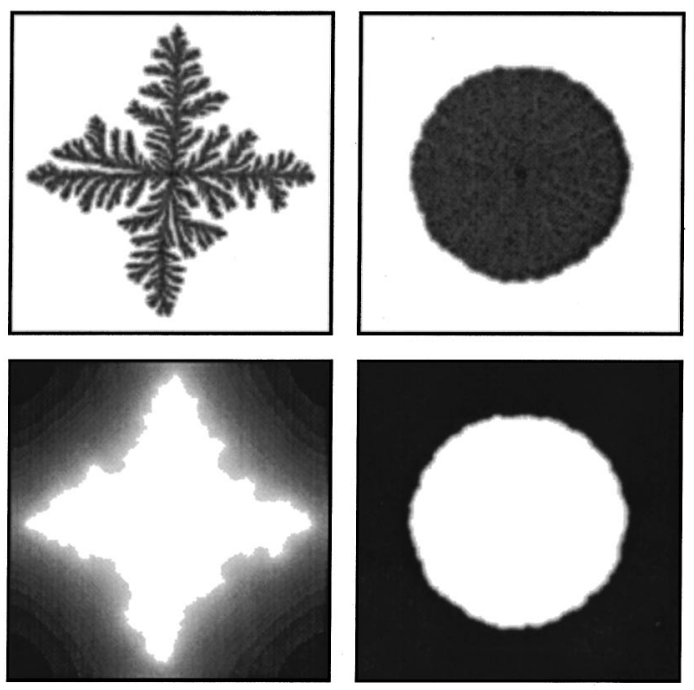

(a)

(b)

FIG. 2. Bacterial colonies (top) and nutrient patterns (bottom) for a fixed value of $D_{0}=0.005$ and two values of initial nutrient: $n_{0}=1$ (a) and $n_{0}=5$ (b). They correspond to times $t=2500$ and 75, respectively.

hardness of the medium, the effective diffusion $D_{0} D_{2} b n$ is still small. The growth is mostly due to reproduction by feeding on the nutrient. For low level of nutrient, i.e., small $n_{0}$, the colony growth is limited by the diffusion of these nutrients. It develops branches, which are thicker as $n_{0}$ increases. The prototype model that reproduces this kind of structure is the diffusion-limited aggregation [12], which is known to form a fractal pattern with a fractal dimension of $d_{F}=1.71$ $[1,22]$. Experiments performed by Matsushita and Fujikawa [9] in region $A$ of Fig. 1 also show a fractal growth with dimension $d_{F}=1.73$ [9]. We have analyzed the fractal nature of the patterns obtained with our model, for $D_{0}=0.005$ and several values of the initial nutrient, from $n_{0}=1$ (DLA-like) to $n_{0}=5$ (rough structure). We have calculated their fractal dimensions by using the box-counting method $[1,22]$. In Fig.

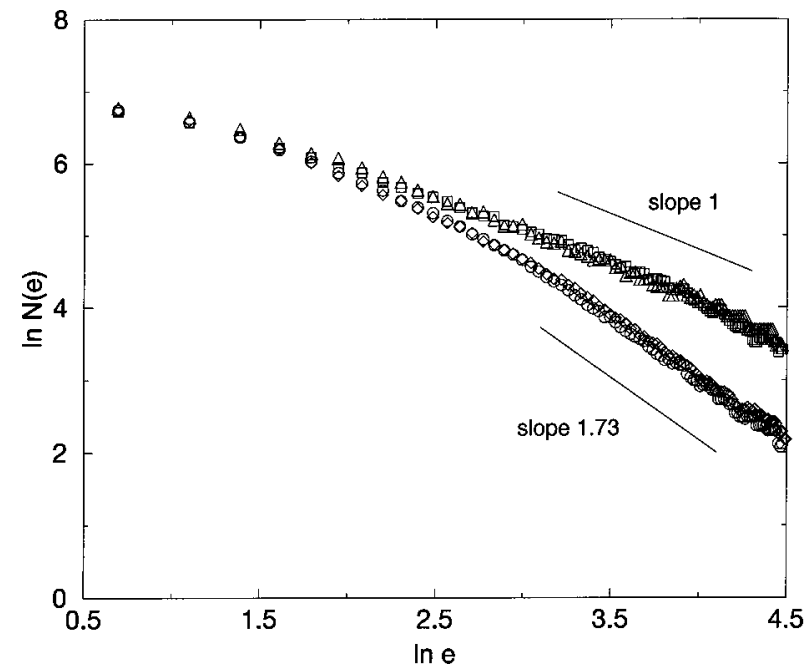

FIG. 3. Fractal dimensions determined by the box-counting method, for $D_{0}=0.005$ and $n_{0}=1(\bigcirc), 2(\diamond), 3(\square)$, and $5(\triangle)$. Two lines of slopes 1 and 1.73 are also plotted for comparison with experiments.

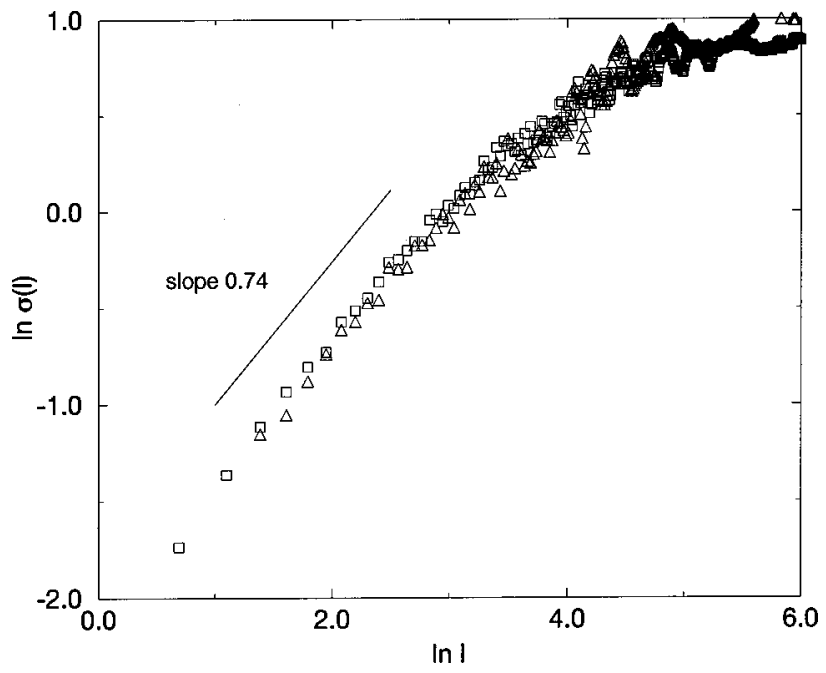

FIG. 4. Width of the rough interface as a function of the length of the interval in which it is measured. They correspond to the pattern of Fig. 2(b) ( $\square$ ) and to a case with the same parameters but with strip geometry $(\triangle)$. A line of slope 0.74 is also plotted for comparison with experimental results.

3 we show, in a $\log$-log plot, the number $N$ of boxes of size $e$ that contain any part of the pattern, versus the size of the boxes. The slopes of the lines represent the fractal dimensions. We observe that the cases that correspond to low nutrient have a fractal dimension of about $d_{F}=1.73$, showing good agreement with experiments. On the other hand, there is an abrupt change between these patterns and those that are not fractal $\left(d_{F}=1\right)$. These last cases can be analyzed in terms of the roughness of their interfaces.

It is well known that Eden structures are not themselves fractal, but their surfaces exhibit a self-affine scaling [22,23]. This implies that, for a long enough time, the width of the rough interface $\sigma$ scales with an exponent $\alpha$ as a function of the length of the interface $l\left(\sigma \sim l^{\alpha}\right)$. The roughness exponent for the Eden model is $\alpha=0.5$. Vicsek et al. [13] analyzed experimental data corresponding to the region $B$ of Fig. 1. They concluded that these colony surfaces are selfaffine with a roughness exponent $\alpha=0.74$. We have checked this point for our dense rough pattern [Fig. 2(b)] by measuring the width $\sigma$ for intervals of interface of length $l$. The results, as a function of $l$, are presented in Fig. 4. In order to avoid additional effects derived from the radial growth of the colony, we have also performed a complementary simulation for the same parameters as Fig. 2(b) but with a strip geometry. To do this, we have used a rectangular lattice of horizontal lateral size $L_{x}=600$, with periodic boundary conditions in the $x$ direction, and taken as an initial condition for bacteria a horizontal line of length $L_{x}$. The results for this case are also plotted in Fig. 4. For both circular and strip cases, we observe analogous behavior to that observed in experiments [13]. Our results show a linear region with a slope compatible with the experimental value $\alpha=0.74$.

With the aim of reproducing other morphologies of Fig. 1, we now keep the initial nutrient fixed at the value $n_{0}=1$ and increase the diffusion parameter $D_{0}$. Results are shown in Figs. 5(a) and 5(b). We observe a crossover from the DLAlike structure [Fig. 2(a)] to a dense branching morphology analogous to that represented in region $E$ of Fig. 1 . 


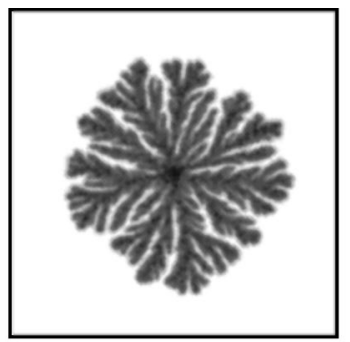

(a)

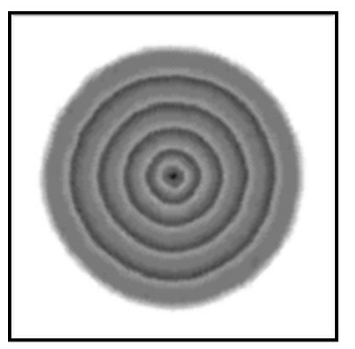

(c)

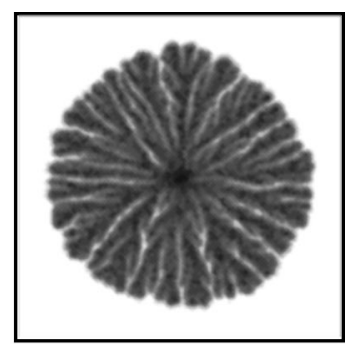

(b)

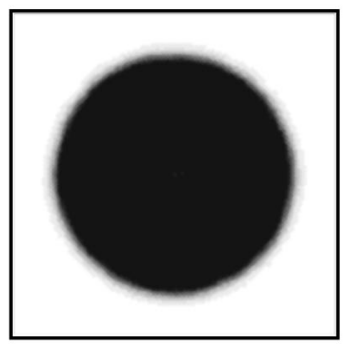

(d)
FIG. 5. Patterns obtained for $n_{0}=1$ with $D_{0}=0.025$ (a) and $D_{0}=0.05$ (b) and for $n_{0}=5$ with $D_{0}=0.05$ (c) and $D_{0}=1$ (d). They correspond to times $t=500,300,50$, and 25 , respectively.

In Fig. 5(c) and 5(d), we present two snapshots obtained for a fixed value of the initial nutrient $n_{0}=5$. They show how different kinds of patterns are obtained when $D_{0}$ is increased: from the dense rough structure [Fig. 2(b)], to concentric rings [Fig. 5(c)], and homogeneous disk [Fig. 5(d)]. They correspond to the regions $B, C$, and $D$, respectively. Homogeneous disks are obtained when $D_{0}$ and $n_{0}$ are so high that the creation term of Eq. (6) is always smaller than $c_{s}$. This means that the value $W_{\max }$, above which the enhanced-movement mechanism begins, is never reached, and bacteria move with the usual diffusion coefficient $D_{0} n$.

Ring patterns correspond to a narrow region of parameters $D_{0}$ and $n_{0}$ for which the creation term of Eq. (6) takes a value between $c_{s}$ and $c_{g}$. As explained in Sec. II, this leads to dynamics in which bacteria move alternatively by the usual diffusion $D_{0} n$ (consolidation phase) or by the enhanced-movement mechanism $D_{0} D_{2} b n$ (migration phase). The two phases are clearly manifested in Fig. 6(a), where we represent the radius of the colony as a function of time. The pattern of concentric rings is a consequence of this dynamic behavior. In Fig. 6(b) we plot the radial density profile, circularly averaged, corresponding to the ring pattern shown in Fig. 5(c). The maxima are formed in the positions where a consolidation phase began. To illustrate this point, we have pointed out in Fig. 6 the positions corresponding to the colony radius at the beginning of each consolidation phase.

Numerically, our model also reproduces the experimentally observed robustness of the growth-plus-consolidation period, which is barely dependent on changes in either nutrient or agar concentrations over a wide range. For high enough $n_{0}$, the value of the global quantity $B$ approaches $n_{0}$. In this limit, as can be derived from Eq. (6) the period is given by

$$
T=D_{0}\left(W_{\max }-W_{\min }\right)\left(\frac{1}{\lambda-D_{0} c_{s}}-\frac{1}{\lambda-D_{0} c_{g}}\right),
$$
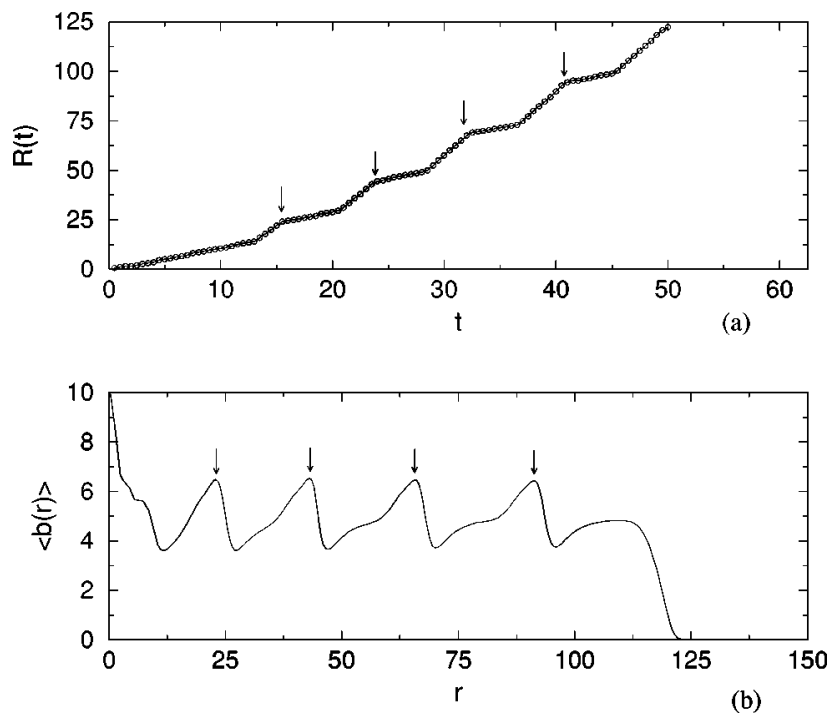

FIG. 6. (a) Time evolution of the colony radius, for parameters $D_{0}=0.05$ and $n_{0}=5$; (b) radial density profile corresponding to time $t=50$ [pattern of Fig. 5(c)]. The positions where each consolidation phase started are pointed out.

which does not depend on $n_{0}$. Moreover, as a function of $D_{0}$, the period also maintains a rather constant value within a certain range (determined by parameters $\lambda, c_{g}$, and $c_{s}$ ) to increase sharply in the boundaries of the ring patterns region $\left(D_{0} \rightarrow \lambda / c_{s}, D_{0} \rightarrow \lambda / c_{g}\right)$. For equal period, the width of the rings increases with $n_{0}$.

\section{CONCLUSIONS}

We have proposed a reaction-diffusion model for the study of bacterial colony growth on agar plates, which consists of two coupled equations for nutrient and bacterial concentrations. The most important feature, which introduces differences from previous models, is the fact that here we consider two mechanisms for the bacterial movement: the random swimming in a liquid medium and a cooperative enhanced movement developed by bacteria when the growth conditions are adverse. The two mechanisms are introduced in our model by means of a diffusion term with two different expressions which depend on the bacterial response to the environmental conditions. This response is modeled as a global variable that presents hysteresis depending on the conditions of the medium. The inhomogeneities of the agar plate have been taken into account as a quenched disorder in the diffusion parameter.

We have shown that, simply by changing the parameters related to the hardness of the medium and the initial nutrient, our model reproduces all the patterns obtained experimentally with the bacterium Bacillus subtilis: DLA-like, denserough disk, DBM-like, ring patterns, and homogeneous disk. We have calculated the fractal dimension of the DLA-like structures and the roughness exponent of the rough disk surface, obtaining results in good agreement with experiments. The ring patterns have been obtained for intermediate values of agar and high nutrient. In this region, the bacterial response presents hysteresis and the two mechanisms of motion work alternatively, leading to cycles of migration and 
consolidation phases. The duration of these cycles is roughly constant for different values of nutrient and agar concentration over a wide range. This periodical dynamics generates patterns of concentric rings.

In summary, the model proposed satisfactorily reproduces the whole experimental morphological diagram. It represents a first attempt at describing the response of bacteria to adverse growth conditions and, in certain conditions, their ability to improve their motility. Further refinements could be made. The bacterial response, here described as a global variable $W(t)$, could be considered in a more realistic way by introducing a coupling term in a local version of Eq. (6) for a field $W(\mathbf{r}, t)$. However, preliminary studies with such a model [24] shows the same essential features previously described.

\section{ACKNOWLEDGMENTS}

We thank I. Ràfols and J.M. Sancho for helpful discussions. This research was supported by the Dirección General de Investigación Científica y Técnica (Spain) (Grant No. PB96-0241-02), by the Comissionat per Universitats i Recerca de la Generalitat de Catalunya (Grant No. SGR97439), and by the Universitat Politècnica de Catalunya (Grant No. PR-9608). We also acknowledge computing support from the Fundació Catalana per a la Recerca and Centre Català de Computació i Comunicacions.
[1] T. Matsuyama and M. Matsushita, in Fractal Geometry in Biological Systems, edited by P.M. Iannaccone and M. Khokha (CRC Press, Boca Raton, 1996).

[2] E. Ben-Jacob, Contemp. Phys. 38, 205 (1997).

[3] E. Ben-Jacob, O. Shochet, A. Tenenbaum, I. Cohen, A. Czirók, and T. Vicsek, Nature (London) 368, 46 (1994).

[4] E.O. Budrene and H.C. Berg, Nature (London) 349, 630 (1991); 376, 49 (1995).

[5] J.A. Shapiro and D. Trubatch, Physica D 49, 214 (1991).

[6] O. Rauprich, M. Matsushita, C.J. Weijer, F. Siegert, S.E. Esipov, and J.A. Shapiro, J. Bacteriol. 178, 6525 (1996).

[7] F.D. Williams and R.H. Schwarzhoff, Annu. Rev. Microbiol. 32, 101 (1978); C. Allison and C. Hughes, Sci. Prog. 75, 403 (1991); R. Belas, D. Erskine, and D. Flaherty, J. Bacteriol. 173, 6279 (1991).

[8] I. Ràfols, M.Sc. thesis, Chuo University, Japan, 1998.

[9] M. Matsushita and H. Fujikawa, Physica A 168, 498 (1990); H. Fujikawa and M. Matsushita, J. Phys. Soc. Jpn. 60, 88 (1991).

[10] M. Ohgiwara, M. Matsushita, and T. Matsuyama, J. Phys. Soc. Jpn. 61, 816 (1992).

[11] H. Fujikawa, Physica A 189, 15 (1992).

[12] T.A. Witten and L.M. Sander, Phys. Rev. Lett. 47, 1400 (1981).
[13] T. Vicsek, M. Cserzö, and V.K. Horváth, Physica A 167, 315 (1990).

[14] E. Ben-Jacob and P. Garik, Nature (London) 343, 523 (1990).

[15] B. Chopard, P. Luthi, and M. Droz, J. Stat. Phys. 76, 661 (1994).

[16] L. Zeiri, O. Younes, S. Efrima, and M. Deutsch, Phys. Rev. Lett. 79, 4685 (1997).

[17] J.D. Murray, Mathematical Biology (Springer-Verlag, Berlin, 1989).

[18] J. Wakita, K. Komatsu, A. Nakahara, T. Matsuyama, and M. Matsushita, J. Phys. Soc. Jpn. 63, 1205 (1994).

[19] K. Kawasaki, A. Mochizuki, M. Matsushita, T. Umeda, and N. Shigesada, J. Theor. Biol. 188, 177 (1997).

[20] M. Matsushita, J. Wakita, H. Itoh, I. Ráfols, T. Matsuyama, H. Sakaguchi, and M. Mimura, Physica A 249, 517 (1998).

[21] E. Esipov and J.A. Shapiro, J. Math. Biol. 36, 249 (1998).

[22] A.L. Barabasi and H.E. Stanley, Fractal Concepts in Surface Growth (Cambridge University Press, Cambridge, England, 1995).

[23] J. Krug and H. Spohn, in Solids Far From Equilibrium, edited by C. Godrèche (Cambridge University Press, Cambridge, England, 1992).

[24] A.M. Lacasta, I.R. Cantalapiedra, C.E. Auguet, A. Peñaranda, and L. Ramírez-Piscina (unpublished). 